\section{Inhibitor MEKanisms}

\section{By Benjamin Boettner, Assistant Editor}

Roche's Genentech Inc. unit has figured out why different MEK inhibitors exhibit varying efficacy against BRAF- or K-Ras-driven cancers, which could help in the design of next-generation inhibitors with improved therapeutic indexes. ${ }^{1}$ Genentech already has one inhibitor for each kind of tumor in clinical trials.

Mutations in the K-Ras (KRAS) and BRAF genes are responsible for overactivation of the Ras-Raf-MEK-MAPK pathway in many cancers. MEK is a key component of this signaling cascade, but for unknown reasons $B R A F$-mutant tumors thus far have been more sensitive than KRAS-mutant tumors to MEK inhibitors. ${ }^{2}$

One hypothesis is that BRAF and KRAS interact differently with MEK. Oncogenic BRAF phosphorylates MEK directly, whereas KRAS acts upstream to activate Raf kinases and other tumorigenic effectors such as phosphoinositide 3-kinase (PI3K).

Consequently, BRAF-mutant cancer cells have higher basal levels of phosphorylated MEK than KRAS-mutant cells, rendering the former more sensitive to MEK inhibitors ${ }^{3}$ (see Figure 1, "Targeting MEK in cancer").

Genentech researchers have now taken a closer look at why three of their inhibitors-cobimetinib (GDC-0973), GDC-0623 and G-573exhibit differential efficacy. The former is most effective in BRAFmutant cancer cell lines, whereas the latter two show better results in KRAS-mutant cells.

GDC-0973 was discovered by Exelixis Inc. and is partnered with Genentech. The molecule is in Phase III trials for melanoma. GDC-0623 and G-573 were discovered at Genentech. GDC-0623, the more potent of the two, is in Phase I testing for solid tumors.

Using a combination of structural, biochemical and physiological data, a team led by Georgia Hatzivassiliou and Marcia Belvin showed that the compounds interacted with MEK in very different ways.

Hatzivassiliou is a scientist and Belvin is associate director of translational oncology at Genentech.

All three compounds are allosteric MEK inhibitors that do not compete with ATP for the kinase's active site but rather bind to an adjacent activation loop. The compounds do so in different ways. GDC-0973 hits a conformation of the loop that is induced by BRAFmediated MEK phosphorylation. GDC-0623 and G-573 bind to unphosphorylated MEK at the Ser-212 residue and prevent MEK phosphorylation by wild-type Raf.

GDC-0973 showed more inhibition than GDC-0623 or G-573 in mouse xenografts of melanoma or colon cancer harboring a mutated $B R A F$ allele. In contrast, GDC-0623 and G-573 showed better results than GDC-0973 in KRAS-mutant pancreatic or lung adenocarcinoma xenografts.

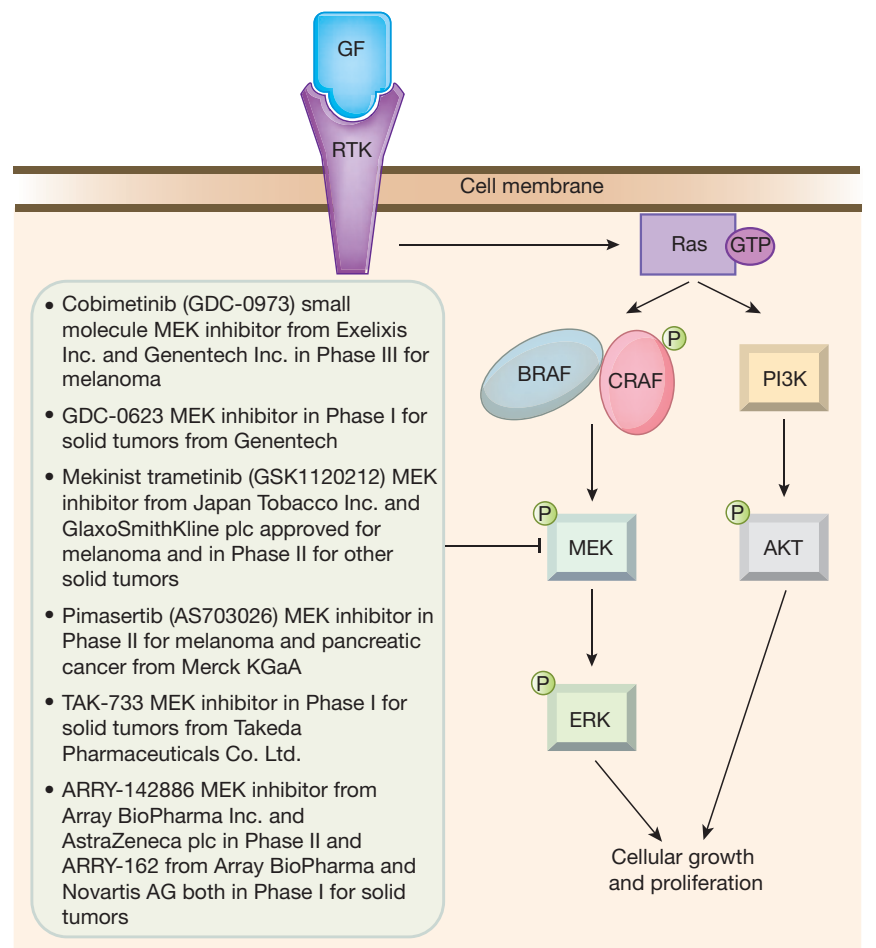

Figure 1. Targeting MEK in cancer. According to findings published in Nature, two classes of MEK inhibitors exhibit different effects in $K$-Ras (KRAS)- and BRAF-mutant backgrounds. In cancer cells, BRAF or Ras proteins often acquire activating mutations that allow them to become independent of growth factor (GF) and receptor tyrosine kinase (RTK) function and to overstimulate their downstream signaling elements. BRAF strongly phosphorylates and activates MEK as its primary downstream target. KRAS activates MEK less strongly than BRAF but also drives phosphoinositide 3-kinase (PI3K) and protein kinase B (PKB; PKBA; AKT; AKT1) signaling. MEK-ERK and PI3K-AKT signaling can cooperate to promote cancer cell proliferation.

In cancer cells that develop resistance to BRAF inhibitors like Zelboraf vemurafenib, BRAF trans-activates wild-type CRAF (RAF1), and the BRAF-CRAF complex is further stimulated by elevated Ras activity. Ras activity can be enhanced by de novo mutation of neuroblastoma Ras viral (v-Ras) oncogene (NRAS) or hyperactive RTKs.

The findings were published in Nature.

According to Hatzivassiliou, the results point to the need for assessing the impact of BRAF and KRAS mutation status on clinical efficacy of individual allosteric MEK inhibitors.

"Conclusions with one inhibitor don't necessarily translate to conclusions about MEK as a target in general. For example, past clinical trials with MEK inhibitors that didn't demonstrate strong clinical efficacy need to be revisited with new compounds with known mechanisms of action," she said.

Kevin Koch, president and CSO of Array BioPharma Inc., told SciBX 
that "this is one of the nicest examples of drug discovery where chemical structure is related to target structure. At a high level there are not many examples where minor changes in drug interactions with their targets are shown to create novel biology resulting in different drug efficacies."

Array's MEK inhibitors for solid tumors include ARRY-142886, which is in Phase II testing and is partnered with AstraZeneca plc, and ARRY-162, which is in Phase I trials and is partnered with Novartis AG.

Levi Garraway, an associate professor at Harvard Medical School, an assistant professor at the Dana-Farber Cancer Institute and a principal investigator at the Broad Institute of MIT and Harvard, said that the study may open up more fine-tuned therapeutic approaches to BRAFand KRAS-driven cancers.

"This preclinical study should definitely heighten interest in clinical trials for MEK inhibitors. There is the potential that distinctions made in the work could eventually lead to cancer drugs that offer additional treatment options to the ones available right now," said Garraway.

\section{Gaining traction}

In addition to helping fine-tune patient selection, the study's mechanistic insights could spur the development of MEK inhibitors with

"This is one of the nicest examples of drug discovery where chemical structure is related to target structure. At a high level there are not many examples where minor changes in drug interactions with their targets are shown to create novel biology resulting in different drug efficacies." -Kevin Koch, Array BioPharma Inc. improved efficacy.

According to Garraway, "The findings reveal new binding mechanisms and may offer a new pharmacological rationale for a subset of MEK inhibitors that could provide a way to get traction in KRAS-mutant cancers. Of course, it will be crucial to find out whether so-called KRAS-selective MEK inhibitors have any activity in patients carrying KRAS-mutant tumors."

Gary Johnson, chair of the Department of Pharmacology at The University of North Carolina at Chapel Hill School of Medicine, told SciBX that "increasing the allosteric selectivity through high-affinity interactions like those described with Ser212 in this study is predicted to ensure greater selectivity of these kinase inhibitors. Moreover, the unique contacts formed may further increase the durability of inhibitor responses by slowing the dissociation of the inhibitor compound from its target kinase."

Koch said that it will be important to explore different MEK inhibitor categories across multiple tumor types.

Another important question is whether inhibitors with efficacy in $K R A S$-mutant cancers can interfere with the growth of BRAF-mutant tumors resistant to Zelboraf vemurafenib, an oral small molecule inhibitor of the oncogenic BRAF V600E. BRAF-mutant cancers that are resistant to BRAF inhibitors could be targeted by MEK inhibitors that are particularly active in a KRAS-mutant background.

For example, Zelboraf resistance arises when mutant BRAF forms a complex with wild-type CRAF (RAF1). CRAF's kinase activity is triggered by Ras overactivity, which originates with de novo mutations of the KRAS homolog neuroblastoma Ras viral ( $v$-Ras) oncogene (NRAS) or elevated receptor tyrosine kinase (RTK) activity $^{4}$ (see Figure 1, “Targeting MEK in cancer").

Roche has rights to Zelboraf from Plexxikon Inc., which Daiichi Sankyo Co. Ltd. acquired in 2011.

Lee Graves told SciBX, "It would make sense to test KRAS-specific MEK inhibitors against BRAF inhibitor-resistant melanomas since they appear to depend on high Ras activity. My prediction is that these may be superior to the MEK inhibitors exhibiting comparatively higher activities in BRAF-mutant backgrounds."

Graves is an associate professor in the Department of Pharmacology at the UNC at Chapel Hill School of Medicine.

Garraway agreed. "The addition of MEK inhibitors with elevated potential in NRAS-mutant conditions may conceivably help prevent escape from mutant BRAF inhibition and the development of resistance," he said.

The authors did not disclose patent and licensing status of the findings described in the Nature paper.

The most advanced MEK inhibitor is Mekinist trametinib (GSK1120212) from GlaxoSmithKline plc. The drug, which GSK in-licensed from Japan Tobacco Inc., is approved for melanoma and is in Phase II testing for other tumor types.

Other MEK inhibitors include Merck KGaA's pimasertib (AS703026), which is in Phase II testing for melanoma and pancreatic cancer, and Takeda Pharmaceutical Co. Ltd.'s TAK-733, which is in Phase I trials for solid tumor indications.

Boettner, B. SciBX 6(36); doi:10.1038/scibx.2013.981

Published online Sept. 19, 2013

\section{REFERENCES}

1. Hatzivassiliou, G. et al. Nature; published online Aug. 11, 2013; doi:10.1038/nature12441

Contact: Marcia Belvin, Genentech Inc., South San Francisco,

Calif.

e-mail: belvin.marcia@gene.com

Contact: Georgia Hatzivassiliou, same affiliation as above e-mail: hatzivassiliou.georgia@gene.com

2. Solit, D.B. et al. Nature 439 , 358-362 (2006)

3. Pratilas, C.A. et al. Proc. Natl. Acad. Sci. USA 106, 4519-4524 (2009)

4. Poulikakos, P.I. \& Rosen, N. Cancer Cell 19, 11-15 (2011)

\section{COMPANIES AND INSTITUTIONS MENTIONED}

Array BioPharma Inc. (NASDAQ:ARRY), Boulder, Colo. AstraZeneca plc (LSE:AZN; NYSE:AZN), London, U.K.

Broad Institute of MIT and Harvard, Cambridge, Mass.

Daiichi Sankyo Co. Ltd. (Tokyo:4568; Osaka:4568), Tokyo, Japan

Dana-Farber Cancer Institute, Boston, Mass.

Exelixis Inc. (NASDAQ:EXEL), South San Francisco, Calif.

Genentech Inc., South San Francisco, Calif.

GlaxoSmithKline plc (LSE:GSK; NYSE:GSK), London, U.K.

Harvard Medical School, Boston, Mass.

Japan Tobacco Inc. (Tokyo:2914; Osaka:2914), Tokyo, Japan

Merck KGaA (Xetra:MRK), Darmstadt, Germany

Novartis AG (NYSE:NVS; SIX:NOVN), Basel, Switzerland

Roche (SIX:ROG; OTCQX:RHHBY), Basel, Switzerland

Takeda Pharmaceutical Co. Ltd. (Tokyo:4502), Osaka, Japan

The University of North Carolina at Chapel Hill School of

Medicine, Chapel Hill, N.C. 